\title{
STABILITY OF FLEXIBLE SPHERICAL PANELS OF VARIABLE THICKNESS UNDER VARIOUS FIXING CONDITIONS
}

\author{
V. V. Chupin, D. E. Chernogubov* \\ Ural Federal University named after the first President of Russia B.N. Yeltsin, 19 Mira st., Ekaterinburg, Russia \\ *Corresponding author. E-mail: d.e.chernogubov@urfu.ru; address for correspondence: 19, ul. Mira, 620002, \\ Ekaterinburg, Russia; tel.: +7 3433754533
}

An algorithm has been developed for studying the stability of elastic thin-shell systems consisting of shells of revolution supported by rings. On the basis of this algorithm, a computer program has been written that allows one to determine the values of critical loads and buckling modes of shells in a wide range of geometrical, physical and power parameters. The stability of spherical panels of variable thickness under different conditions of fixing has been studied.

Keywords: shell, stability, critical load.

DOI: $10.17804 / 2410-9908.2015 .5 .045-057$

\section{References}

1. Valishvili N.V. Metody rascheta obolochek vrashcheniya na ETSVM [Methods for Calculating Shells of Revolution on a Computer]. M., Mashinostroenie Publ., 1976, 278 p. (In Russian).

2. Volmir A.S. Ustoichivost deformiruemyh sistem [The Stability of Deformable Systems]. M., Nauka Publ., 1976, 984 p. (In Russian).

3. Godunov S.K. On the numerical solution of boundary value problems for systems of ordinary differential equations. Uspekhi Matematicheskikh Nauk, 1961, no. 16, pp. 171-174. (In Russian).

4. Grigolyuk E.I., Shalashilin V.I. Problemy nelineinogo deformirovaniya [Problems of Nonlinear Deformation]. M., Nauka Publ., 1988, 231 p. (In Russian).

5. Grigorenko Ya.M. Izotropnye $i$ anizotropnye sloistye obolochki vrashcheniya peremennoi zhestkosti [Isotropic and Anisotropic Laminated Shells of Revolution of Variable Rigidity]. Kiev, Naukova Dumka Publ., 1973, 228 p. (In Russian).

6. Grigorenko Ya.M., Kryukov N.N. Solving nonlinear boundary value problems of the statics of flexible laminated shells in the supercritical region. Soviet Applied Mechanics, 1983, Vol. 19, iss. 3, pp 217-221.

7. Emelyanov I.G. Kontaktnye zadachi teorii obolochek [Contact Problems of the Theory of Shells]. Ekaterinburg, UrO RAN, 2009, 184 p. (In Russian).

8. Kantorovich L.V., Akilov G.R. Funktsionalnyi analiz v normirovannykh prostranstvakh [Functional Analysis in Normed Spaces]. M., Fizmatgiz Publ., 1959, 684 p. (In Russian).

9. Karmishin A.V., Lyaskovets V.A., Myachenkov V.I., Frolov A.N. Statika $i$ dinamika tonkostennykh obolochechnykh konstruktsiy [Statics and Dynamics of Thin-Walled Shell Structures]. M., Mashinostroenie Publ., 1975, 376 p. (In Russian).

10. Klimanov V.I., Chupin V.V. Statika i Ustoichivost gibkikh neodnorodnykh obolochechnykh sistem [Statics and Stability of Flexible Shell Heterogeneous Systems]. Krasnoyarsk, KrasGU Publ., 1986, 182 p. (In Russian).

11. Korovaitsev A.V. An algorithm for studying the state of shallow shells of revolution under axisymmetric large displacements. Izvestiya vuzov. Mashinostroenie, 1981, iss. 10, pp. 12-15. (In Russian).

12. Mushtari Kh.M., Galimov K.Z. Nelineynaya teoriya uprugikh obolochek [The Nonlinear Theory of Elastic Shells]. Kazan, Tatknigoizdat Publ., 1957, 431 p. (In Russian).

13. Myachenkov V.I., Grigoriev I.V. Raschet obolochechnykh konstruktsiy na EVM [Calculation of 
open-9cCess journal

Shell Structures on a Computer]. M., Mashinostroenie Publ., 1981, 216 p. (In Russian).

14. Novozhilov V.V. Osnovy nelineynoy teorii uprugosti [Fundamentals of Nonlinear Elasticity]. M., Gostekhizdat Publ., 1948, 211 p. (In Russian).

15. Reissner E. Linear and nonlinear theory of shells. In: Tonkostennye obolochechnye konstruktsii [Thin-Walled Shell Structures]. M., Mashinostroenie Publ., 1980, pp. 55-69. (In Russian).

16. Chupin V.V., Chernogubov D.E. Stability of elastic composite shell structures under axisymmetric loading. In: Stroitelstvo $i$ obrazovanie [Civil Engineering and Education]. Ekaterinburg, UrFU, 2011, iss. 14, pp. 29-32. (In Russian).

17. Shapovalov L.A. Equations of thin elastic shells for asymmetric deformations. Mechanics of Solids, 1976, iss. 3, pp. 62-72. 
Подана в журнал: 23.06 .2015

УДК 539.3

DOI: $10.17804 / 2410-9908.2015 .5 .045-057$

\title{
УСТОЙЧИВОСТЬ ГИБКИХ СФЕРИЧЕСКИХ ПАНЕЛЕЙ ПЕРЕМЕННОЙ ТОЛЩИНЫ ПРИ РАЗЛИЧНЫХ УСЛОВИЯХ ЗАКРЕПЛЕНИЯ
}

\author{
В. В. Чупин, Д. Е. Черногубов*
}

Федеральное государственное автономное образовательное учреждение высшего профессионального образования «Уральский федеральный университет имени первого Президента России Б.Н. Ельцина», ул. Мира, 19, Екатеринбург, Россия

*Ответственный автор. Электронная почта: d.e.chernogubov@urfu.ru; адрес для переписки: ул. Мира, 19, 620002, Екатеринбург, Россия; телефон: +7 (343) 375-45-33

Разработан алгоритм исследования устойчивости упругих тонкостенных оболочечных систем, состоящих из оболочек вращения подкрепленных кольцами. На основе данного алгоритма написана программа для ЭВМ, позволяющая определять величины критических нагрузок и формы потери устойчивости оболочек в широком диапазоне изменения геометрических, физических и силовых параметров. Исследована устойчивость сферических панелей переменной толщины при различных условиях закрепления.

Ключевые слова: оболочка, устойчивость, критическая нагрузка.

\section{1. Введение}

Для исследования устойчивости гибких оболочек широко используется статический критерий Эйлера [2,9]. При этом критическая нагрузка определяется как наименьшая из нагрузок, при которых появляются смежные формы равновесия. Таким образом, приходим к задаче определения собственных значений системы линеаризованных дифференциальных уравнений, которые получаются из нелинейных уравнений при рассмотрении двух близких смежных равновесных состояний и содержат докритические осесимметричные усилия и деформации.

Большинство работ посвящено исследованию гибких составных оболочек вращения в квадратичном приближении (средний изгиб), в которых предложены эффективные алгоритмы для широкого класса задач, основаны на сведении нелинейной краевой задачи к суперпозиции линейных задач Коши [9].

Для решения нелинейных краевых задач теории оболочек широкое распространение в последнее время получил метод линеаризации Ньютона-Канторовича [8], обладающий квадратичной сходимостью. Идея этого метода заключается в линеаризации системы дифференциальных уравнений, условий сопряжения оболочек, граничных условий и в построении итерационного процесса решения нелинейной краевой задачи, для которого задается начальное приближение, и на каждом этапе решается линейная краевая задача.

При исследовании задач о деформировании оболочек в линейной постановке исходная система дифференциальных уравнений сводится к ряду задач Коши, каждая из которых интегрируется одним из численных методов Рунге-Кутта, Кутта-Мерсона, Адамса и другими. Однако вследствие жесткости дифференциальных уравнений теории оболочек возникают проблемы с обеспечением устойчивости вычислительного процесса. Для преодоления указанных трудностей используется получивший широкое распространение метод дискретной ортогонализации С.К. Годунова [3].

К линейным задачам статики несимметрично нагруженных упругих составных оболочек вращения этот подход применен Я.М. Григоренко [5], И.Г. Емельяновым [7].

Высокая эффективность этого метода решения задач среднего изгиба для 
осесимметрично нагруженных составных оболочек вращения подтверждена в работах В.И. Мяченкова, А.Н. Фролова и др. [9], Я.М. Григоренко, Н.Н. Крюкова [6].

При исследовании закритических деформаций в задачах среднего изгиба оболочек трудности, возникающие с прохождением особых точек (предельные точки на кривой деформирования), устраняются с помощью введения дополнительных уравнений для вспомогательных параметров и путем смены в окрестности особых точек ведущего параметра на другой, имеющий в окрестности этой точки монотонный характер [1]. Для построения кривой деформирования нагрузка-прогиб в области закритических деформаций применительно к пологой сферической оболочке данный прием использован в работах Я.М. Григоренко, Н.Н. Крюкова [6], А.В. Коровайцева [11].

Развитию метода продолжения по параметру и исследованию им оболочек, круговых арок, посвящена монография Э.И. Григолюка, В.И. Шалашилина [4].

Задача определения критических нагрузок при осесимметричном нагружении приводит к решению однородных линейных краевых задач для отдельных гармоник.

Для составных оболочек вращения при осесимметричном нагружении самое широкое применение в задачах устойчивости находит метод сведения линейной краевой задачи к суперпозиции линейных задач Коши. На основе этого метода В.И. Мяченковым и др. [9, 13] приведены решения для большого количества составных оболочечных конструкций. В них рассмотрены оболочки с кольцами, исследовано влияние начальных прогибов, действие температуры, комбинированного нагружения.

В данной работе используются уравнения сильного изгиба оболочки, т. е. докритические осесимметричные усилия и деформации определяются из решения геометрически нелинейной задачи для оболочки, испытывающей сильный изгиб [12, 14]; прогибы оболочек сравнимы с характерными размерами оболочек, а более строго это означает, что компоненты линейной деформации малы по сравнению с единицей, а на величины углов поворота нормали к срединной поверхности не накладывается никаких ограничений.

Рассмотрение уравнений, описывающих сильный изгиб оболочек, проводится в теоретических работах $[12,15,17]$.

При сильном изгибе исходные нелинейные разрешающие уравнения содержат члены с сильной нелинейностью. Для решения этих уравнений используем метод линеаризации Ньютона-Канторовича [8] и используем метод последовательных нагружений для организации шагового процесса и смены ведущего параметра в окрестности особых точек.

Для определения критических нагрузок составных оболочек вращения при сильном осесимметричном изгибе используем метод сведения линейной краевой задачи к суперпозиции линейных задач Коши и метод дискретной ортогонализации С.К. Годунова.

\section{2. Задача исследования устойчивости составной упругой оболочечной системы}

Критические нагрузки для составной упругой оболочечной конструкции будем искать на основе статического критерия Эйлера [2]. Это позволяет свести отыскание критической нагрузки к нахождению собственного значения системы линеаризованных дифференциальных уравнений, которые получаются из нелинейных уравнений при рассмотрении двух смежных равновесных состояний.

Предположим, что тонкостенная конструкция, составленная из оболочек вращения на (m-1) этапе нагружения, находится в устойчивом осесимметричном состоянии и при добавлении малой нагрузки в процессе т-го этапа нагружения стремится перейти в смежное неосесимметричное состояние равновесия.

Напряженно-деформированное состояние, предшествующее моменту потери устойчивости для конструкции, составленной из оболочек вращения, испытывающей сильный изгиб, определяется с помощью алгоритмов, изложенных в работах $[10,13]$.

Обозначим величины, характеризующие осесимметричное напряженно- 
деформированное состояние гибкой оболочки после (m-1) этапа нагружения, следующим образом: $N_{i}^{0}, Q_{s}^{0}, M_{i}^{0}, u^{0}, w^{0}, \varphi^{0}, r, \ldots$, где $N_{i}^{0}$ - нормальные усилия; $Q_{s}^{0}$ - поперечное усилие; $M_{i}^{0}-$ изгибающие моменты; $u^{0}-$ касательные перемещения; $w^{0}-$ нормальные перемещения; $\varphi^{0}-$ угол, образованный нормалью к координатной поверхности и осью вращения; $r$ - радиус параллельного круга; 0 - указывает на осесимметричное состояние; $i=s, \theta$.

За счет малых неосесимметричных добавок $N_{i}, Q_{s}, M_{i}, u, v, w, \ldots$ возможен переход от осесимметричного к неосесимметричному деформированному состоянию, для которого искомые величины представим в виде сумм:

$$
\begin{array}{lll}
\tilde{N}_{x}=N_{x}^{0}+N_{x} ; & \tilde{N}_{z}=N_{z}^{0}+N_{z} ; & \tilde{\hat{S}}=\hat{S}^{0}+\hat{S} ; \\
\tilde{M}_{s}=M_{s}^{0}+M_{s} ; & \widetilde{u}_{x}=u_{x}^{0}+u_{x} ; & \tilde{u}_{z}=u_{z}^{0}+u_{z} ; \\
\widetilde{v}=v^{0}+v ; & \widetilde{r}=r+u_{x} ; & \widetilde{z}=z^{0}+u_{z} ; \\
\widetilde{\varphi}=\varphi+\theta_{s}, & &
\end{array}
$$

где $\hat{S}^{0}=v^{0}=0$, так как (m-1) состояние равновесия является осесимметричным.

Полагая, что неосесимметричное деформированное состояние мало отличается по своей геометрии от смежного осесимметричного равновесного состояния, можно принять коэффициенты Ляме равными $\widetilde{A}_{s}=1, \widetilde{A}_{\theta}=\widetilde{r}$.

Уравнения равновесия запишем в декартовой системе координат в виде [10]:

$$
\begin{aligned}
& \frac{\partial \tilde{N}_{x}}{\partial \widetilde{S}}=-\frac{\cos \widetilde{\varphi}}{\widetilde{r}} \widetilde{N}_{x}+\left(\frac{\cos ^{2} \widetilde{\varphi}}{\widetilde{r}}+\frac{\sin \widetilde{\varphi}}{\widetilde{R}_{\theta}}\right) \tilde{N}_{\theta}-\frac{\cos \tilde{\varphi}}{\widetilde{r}} \frac{\partial \tilde{\hat{S}}}{\partial \theta}-\frac{\sin \widetilde{\varphi}}{\widetilde{r}^{2}} \frac{\partial^{2} \tilde{M}_{\theta}}{\partial \theta^{2}}-\widetilde{q}_{x} ; \\
& \frac{\partial \widetilde{N}_{z}}{\partial \widetilde{S}}=-\frac{\cos \widetilde{\varphi}}{\widetilde{r}} \widetilde{N}_{z}-\frac{\sin \widetilde{\varphi}}{\widetilde{r}} \frac{\partial \hat{\hat{S}}}{\partial \theta}+-\frac{\cos \widetilde{\varphi}}{\widetilde{r}^{2}} \frac{\partial^{2} \widetilde{M}_{\theta}}{\partial \theta^{2}}+\frac{2}{\widetilde{r}^{2}} \frac{\partial \widetilde{H}}{\partial \theta}+\cos \widetilde{\varphi}\left(\frac{\sin \widetilde{\varphi}}{\widetilde{r}}-\frac{1}{\widetilde{R}_{\theta}}\right) \widetilde{N}_{x}-\widetilde{q}_{z} ; \\
& \frac{\partial \widetilde{\widetilde{S}}}{\partial \widetilde{S}}=-\frac{2 \cos \widetilde{\varphi}}{\widetilde{r}} \widetilde{\widetilde{S}}-\frac{1}{\widetilde{r}} \frac{\partial \widetilde{N}_{\theta}}{\partial \theta}-\frac{\sin \widetilde{\varphi}}{\widetilde{r}^{2}} \frac{\partial \tilde{M}_{\theta}}{\partial \theta} \\
& \frac{\partial \widetilde{M}_{s}}{\partial \widetilde{s}}=-\frac{\cos \widetilde{\varphi}}{\widetilde{r}}\left(\tilde{M}_{s}-\tilde{M}_{\theta}\right)-\frac{2}{\widetilde{r}} \frac{\partial \widetilde{H}}{\partial \theta}+\sin \tilde{\varphi} \tilde{N}_{x}-\cos \widetilde{\varphi} \tilde{N}_{z}-\widetilde{m}_{s} .
\end{aligned}
$$

Вычитая из уравнений (2) уравнения равновесия осесимметричного устойчивого состояния, получим для неосесимметричных составляющих дополнительные уравнения равновесия:

$$
\begin{aligned}
& \frac{\partial N_{x}}{\partial s}=-\frac{\cos \varphi}{r} N_{x}+\frac{1}{r} N_{\theta}-\frac{\cos \varphi}{r} \frac{\partial \hat{S}}{\partial \theta}-\frac{\sin \varphi}{r^{2}} \frac{\partial^{2} M_{\theta}}{\partial \theta^{2}}+\left[\frac{\sin \varphi}{r} N_{x}^{0} \theta_{s}+\sin \varphi N_{\theta}^{0} \bar{\chi}_{\theta}^{k \neq 0}\right] \\
& \frac{\partial N_{z}}{\partial s}=-\frac{\cos \varphi}{r} N_{z}-\frac{\sin \varphi}{r} \frac{\partial \hat{S}}{\partial \theta}+\frac{\cos \varphi}{r^{2}} \frac{\partial^{2} M_{\theta}}{\partial \theta^{2}}+\frac{2}{r^{2}} \frac{\partial H}{\partial \theta}+\left[\frac{\sin \varphi}{r} N_{z}^{0} \theta_{s}-\cos \varphi N_{\theta}^{0} \bar{\chi}_{\theta}^{k \neq 0}\right]
\end{aligned}
$$

$\frac{\partial \widehat{S}}{\partial s}=-\frac{2 \cos \varphi}{r} \widehat{S}-\frac{1}{r} \frac{\partial N_{\theta}}{\partial \theta}-\frac{\sin \varphi}{r^{2}} \frac{\partial M_{\theta}}{\partial \theta} ;$

$\frac{\partial M_{s}}{\partial \widetilde{s}}=-\frac{\cos \varphi}{r}\left(M_{s}-M_{\theta}\right)-\frac{2}{r} \frac{\partial H}{\partial \theta}+\sin \varphi N_{x}-\cos \varphi N_{z}+\left[\frac{\sin \varphi}{r}\left(M_{s}^{0}-M_{\theta}^{0}\right) \theta_{s}+\sin \varphi N_{x}^{0} \theta_{s}-\cos \varphi N_{z}^{0} \theta_{s}\right]$, 
где $\bar{\chi}_{\theta}^{k \neq 0}=\left\{\begin{array}{l}0, \quad n p u \quad k=0 \\ \bar{\chi}_{\theta} \equiv \frac{1}{\widetilde{R}_{\theta}}-\frac{\sin \widetilde{\varphi}}{\widetilde{r}}=\chi_{\theta}-\frac{\cos \widetilde{\varphi}}{\widetilde{r}} \widetilde{\theta_{s}}, \quad \text { npu } k \neq 0\end{array} ;\right.$

$\chi_{\theta}-$ изменение кривизны при переходе к несимметричному состоянию; $\theta_{s}-$ угол поворота нормали.

Уравнения записаны для деформированного состояния оболочек и содержат докритические осесимметричные усилия $N_{x}^{0}, N_{z}^{0}, M_{s}^{0}, M_{\theta}^{0}$ (знак тильды для этих величин и для $\varphi, r, R_{s}, R_{\theta}$ опущен). Докритическое изменение геометрии оболочки учитывается в них за счет использования геометрии деформированной поверхности.

Дополнительные уравнения равновесия (3) в гауссовой системе координат, связанной с оболочкой, имеют вид:

$$
\begin{aligned}
& \frac{\partial N_{s}}{\partial s}=-\frac{\cos \varphi}{r}\left(N_{s}-N_{\theta}\right)-\frac{1}{R_{s}} \widehat{Q}_{s}-\frac{1}{r} \frac{\partial \widehat{S}}{\partial \theta}+\frac{2 \sin \varphi}{r^{2}} \frac{\partial H}{\partial \theta}+\frac{\sin \varphi}{r} N_{s}^{0} \theta_{s} ; \\
& \frac{\partial \widehat{Q}_{s}}{\partial s}=-\frac{\cos \varphi}{r} \widehat{Q}_{s}+\frac{N_{s}}{R_{s}}+\frac{N_{\theta}}{R_{\theta}}-\frac{1}{r^{2}} \frac{\partial^{2} M_{\theta}}{\partial \theta^{2}}-\frac{2 \cos \varphi}{r^{2}} \frac{\partial H}{\partial \theta}+\frac{\sin \varphi}{r} Q_{s}^{0} \theta_{s}+N_{\theta}^{0} \bar{\chi}_{\theta}^{k \neq 0} ; \\
& \frac{\partial \hat{S}}{\partial s}=-\frac{2 \cos \varphi}{r} \widehat{S}-\frac{1}{r} \frac{\partial N_{\theta}}{\partial \theta}-\frac{\sin \varphi}{r^{2}} \frac{\partial M_{\theta}}{\partial \theta} ; \\
& \frac{\partial M_{s}}{\partial s}=-\frac{\cos \varphi}{r}\left(M_{s}-M_{\theta}\right)-\frac{2}{r} \frac{\partial H}{\partial \theta}+\widehat{Q}_{s}+N_{s}^{0} \theta_{s}+\frac{\sin \varphi}{r}\left(M_{s}^{0}-M_{\theta}^{0}\right) \theta_{s} .
\end{aligned}
$$

Запишем геометрические соотношения для дополнительных перемещений и деформаций в декартовой системе координат [10]:

$$
\begin{aligned}
& \frac{\partial u_{x}}{\partial s}=\cos \varphi \varepsilon_{s}-\sin \varphi \theta_{s} ; \\
& \frac{\partial u_{z}}{\partial s}=\sin \varphi \varepsilon_{s}+\cos \varphi \theta_{s} ; \\
& \frac{\partial v}{\partial s}=\varepsilon_{s \theta}-\frac{1}{r} \frac{\partial}{\partial \theta}\left(\cos \varphi u_{x}+\sin \varphi u_{z}\right)+\frac{\cos \varphi}{r} v ; \\
& \frac{\partial \theta_{s}}{\partial s}=\chi_{s} .
\end{aligned}
$$

Эти соотношения, записанные в гауссовой системе координат, принимают форму

$$
\begin{aligned}
& \frac{\partial u}{\partial s}=\varepsilon_{s}-\frac{w}{R_{s}} \\
& \frac{\partial w}{\partial s}=\frac{u}{R_{s}}-\theta_{s} ; \\
& \frac{\partial v}{\partial s}=\varepsilon_{s \theta}-\frac{1}{r} \frac{\partial u}{\partial \theta}+\frac{\cos \varphi}{r} v ; \\
& \frac{\partial \theta_{s}}{\partial s}=\chi_{s} .
\end{aligned}
$$

Дополнительные деформации через дополнительные усилия для смежного напряженного состояния получим, вычитая соотношения упругости осесимметричного состояния из соотношений неосесимметричного (коэффициенты $d_{i j}$ приведены в [5]):

$$
\begin{aligned}
& \varepsilon_{s}=d_{11} N_{s \Delta}+d_{12} M_{s}+d_{13} \varepsilon_{\theta}+d_{14} \chi_{\theta} ; \\
& \varepsilon_{s \theta}=d_{21} \hat{S}+d_{22} \hat{\chi}_{s \theta} ; \\
& \chi_{s}=d_{31} N_{s \Delta}+d_{32} M_{s}+d_{33} \varepsilon_{\theta}+d_{34} \chi_{\theta} ; \\
& N_{\theta}=d_{41} N_{s \Delta}+d_{42} M_{s}+d_{43} \varepsilon_{\theta}+d_{44} \chi_{\theta} ; \\
& M_{\theta}=d_{51} N_{s \Delta}+d_{52} M_{s}+d_{53} \varepsilon_{\theta}+d_{54} \chi_{\theta} ; \\
& H=d_{61} S+d_{62} \hat{\chi}_{s \theta},
\end{aligned}
$$


open-recess journal

где

$$
\begin{aligned}
& N_{s \Delta}=N_{s}-Q_{s}^{0} \theta_{s} ; \quad Q_{s}^{0}=\sin \varphi N_{x}^{0}-\cos \varphi N_{z}^{0} ; \\
& \varepsilon_{\theta}=\frac{1}{r} \frac{\partial v}{\partial \theta}+\frac{u_{x}}{r}=\frac{1}{r}\left(\frac{\partial v}{\partial \theta}+u \cos \varphi+w \sin \varphi\right) ; \\
& \hat{\chi}_{s \theta}=\frac{1}{r^{2}} \frac{\partial u_{z}}{\partial \theta}+\frac{1}{r} \frac{\partial \theta_{s}}{\partial \theta}=-\frac{\sin \varphi}{r^{2}} u+\frac{\cos \varphi}{r^{2}} w+\frac{1}{r} \frac{\partial \theta_{s}}{\partial \theta} ;
\end{aligned}
$$

$\chi_{\theta}=\frac{1}{r^{2}} \frac{\partial^{2}}{\partial \theta^{2}}\left(u_{x} \sin \varphi-u_{z} \cos \varphi\right)+\frac{\sin \varphi}{r^{2}} \frac{\partial v}{\partial \theta}+\frac{\cos \varphi}{r} \theta_{s}=-\frac{1}{r^{2}} \frac{\partial^{2} w}{\partial \theta^{2}}+\frac{\sin \varphi}{r^{2}} \frac{\partial v}{\partial \theta}+\frac{\cos \varphi}{r} \theta_{s}$.

Здесь $\varepsilon_{s}, \varepsilon_{\theta}$ - изменение линейной деформации, а $\chi_{s}, \chi_{\theta}-$ изменение кривизны при переходе оболочки в неосесимметричное состояние.

Разложим переменные в ряды Фурье по окружной координате $\theta$ :

$$
\begin{aligned}
& \left\{N_{s}, Q_{s}, N_{x}, N_{z}, M_{s}, u, w, u_{x}, u_{z}, \theta_{s}\right\}= \\
& \quad=\sum_{k=0}^{\infty}\left\{N_{s}^{k}, Q_{s}^{k}, N_{x}^{k}, N_{z}^{k}, M_{s}^{k}, u^{k}, w^{k}, u_{x}^{k}, u_{z}^{k}, \theta_{s}^{k}\right\} \cos k \theta, \\
& \{\hat{S}, v\}=\sum_{k=0}^{\infty}\left\{\hat{S}^{k}, v^{k}\right\} \sin k \theta,
\end{aligned}
$$

где $k$ - номер гармоники в ряде Фурье.

Разрешающую систему в декартовой системе координат для упругих оболочек образуем, объединив уравнения равновесия (3) и геометрические уравнения (5). Подставляя в нее разложения (8) получим независимые системы уравнений для каждого номера гармоники. Опуская индексы у членов разложения, запишем систему восьми обыкновенных дифференциальных уравнений первого порядка для k-ой гармоники:

$$
\begin{gathered}
\frac{d N_{x}}{d t}=\gamma(t)\left\{-\frac{\cos \varphi}{r} N_{x}+\frac{1}{r} N_{\theta}-\frac{k \cos \varphi}{r} \hat{S}+\frac{k^{2} \sin \varphi}{r^{2}} M_{\theta}+\left[\frac{\sin \varphi}{r} N_{x}^{0} \theta_{s}+\sin \varphi N_{x}^{0} \bar{\chi}_{\theta}^{k \neq 0}\right]\right\} \\
\frac{d N_{z}}{d t}=\gamma(t)\left\{-\frac{\cos \varphi}{r} N_{z}-\frac{k \sin \varphi}{r} \hat{S}-\frac{k^{2} \cos \varphi}{r^{2}} M_{\theta}+\frac{2 k}{r^{2}} H+\left[\frac{\sin \varphi}{r} N_{z}^{0} \theta_{s}-\cos \varphi N_{\theta}^{0} \bar{\chi}_{\theta}^{k \neq 0}\right]\right\} \\
\frac{d \hat{S}}{d t}=\gamma(t)\left\{\frac{k}{r} N_{\theta}-\frac{2 \cos \varphi}{r} \hat{S}+\frac{k \sin \varphi}{r^{2}} M_{\theta}\right\} \\
\frac{d M_{s}}{d t}=\gamma(t)\left\{-\frac{\cos \varphi}{r}\left(M_{s}-M_{\theta}\right)-\frac{2 k}{r} H+\sin \varphi N_{x}-\cos \varphi N_{z}+\right. \\
\left.+\left[\frac{\sin \varphi}{r}\left(M_{s}^{0}-M_{\theta}^{0}\right) \theta_{s}+\cos \varphi N_{x}^{0} \theta_{s}+\sin \varphi N_{z}^{0} \theta_{s}\right]\right\}
\end{gathered}
$$

$$
\begin{aligned}
& \frac{d u_{x}}{d t}=\gamma(t)\left[\cos \varphi \varepsilon_{s}-\sin \varphi \theta_{s}\right] \\
& \frac{d u_{z}}{d t}=\gamma(t)\left[\sin \varphi \varepsilon_{s}+\cos \varphi \theta_{s}\right] \\
& \frac{d v}{d t}=\gamma(t)\left[\varepsilon_{s \theta}+\frac{k}{r}\left(\cos \varphi u_{x}+\sin \varphi u_{z}\right)+\frac{\cos \varphi}{r} v\right] ; \\
& \frac{d \theta_{s}}{d t}=\gamma(t) \chi_{s},
\end{aligned}
$$


open-aceess journal

где

$$
\begin{aligned}
& \gamma(t)=\frac{d s}{d t}=\sqrt{\left(\frac{d r}{d t}\right)^{2}+\left(\frac{d z}{d t}\right)^{2}} ; \\
& \varepsilon_{\theta}=\frac{k}{r} v+\frac{u_{x}}{r} v, \quad \hat{\chi}_{s \theta}=\frac{k}{r^{2}} u_{z}-\frac{k}{r} \theta_{s} ; \\
& \chi_{\theta}=-\frac{k^{2}}{r^{2}}\left(\sin \varphi u_{x}-\cos \varphi u_{z}\right)+\frac{k \sin \varphi}{r^{2}} v+\frac{\cos \varphi}{r^{2}} \theta_{s} ; \\
& t-\text { параметр, который позволяет более удобно задавать форму меридиана }
\end{aligned}
$$
оболочки вращения.

Используя соотношения упругости в форме (7) и выражения (10), приведем (9) к следующей разрешающей системе обыкновенных однородных дифференциальных уравнений восьмого порядка в декартовой системе координат в замкнутой форме:

$$
\frac{d \bar{N}}{d t}=\gamma(t)\left[A_{0}(t)+A_{1}^{m}\left(\bar{N}^{0}, t\right)\right] \bar{N},
$$

где $\bar{N}=\left\{N_{x}, N_{z}, \hat{S}, M_{s}, u_{x}, u_{z}, v, \theta_{s}\right\} ; \quad \bar{N}^{0}$ - вектор-функция, соответствующая докритическому состоянию; $A_{0}, A_{1}$ - квадратные матрицы восьмого порядка [10].

Систему разрешающих однородных уравнений в гауссовой системе координат для упругих оболочек образуем, объединив уравнения равновесия (4) и геометрические соотношения (6). С учетом разложений (8) она распадается на независимые системы уравнений для каждого номера гармоники. Запишем для k-й гармоники систему восьми обыкновенных дифференциальных уравнений первого порядка:

$$
\begin{aligned}
& \frac{d N_{s}}{d s}=-\frac{\cos \varphi}{r}\left(N_{s}-N_{\theta}\right)-\frac{1}{R_{s}} \hat{Q}_{s}-\frac{k}{r} \hat{S}+\frac{2 k \sin \varphi}{r^{2}} H+\frac{\sin \varphi}{r} N_{s}^{0} \theta_{s} \\
& \frac{d \hat{Q}_{s}}{d s}=-\frac{\cos \varphi}{r} \hat{Q}_{s}+\frac{N_{s}}{R_{s}}+\frac{N_{\theta}}{R_{\theta}}-\frac{k^{2}}{r^{2}} M_{\theta}-\frac{2 k \cos \varphi}{r^{2}} H+\frac{\sin \varphi}{r} Q_{s}^{0} \theta_{s}+N_{\theta}^{0} \bar{\chi}_{\theta}^{k \neq 0} ; \\
& \frac{d \hat{S}}{d s}=-\frac{2 \cos \varphi}{r} \hat{S}+\frac{k}{r} N_{\theta}+\frac{k \sin \varphi}{r^{2}} M_{\theta} ; \\
& \frac{d M_{s}}{d s}=-\frac{\cos \varphi}{r}\left(M_{s}-M_{\theta}\right)-\frac{2}{r} \frac{\partial H}{\partial \theta}+\hat{Q}_{s}+N_{s}^{0} \theta_{s}+\frac{\sin \varphi}{r}\left(M_{s}^{0}-M_{\theta}^{0}\right) \theta_{s} ; \\
& \frac{d u}{d s}=\varepsilon_{s}-\frac{w}{R_{s}} ; \\
& \frac{d w}{d s}=\frac{u}{R_{s}}-\theta_{s}^{m} ; \\
& \frac{d v}{d s}=\varepsilon_{s \theta}+\frac{k}{r} u+\frac{\cos \varphi}{r} v ; \\
& \frac{d \theta_{s}}{d s}=\chi_{s} .
\end{aligned}
$$

Здесь индексы у членов разложения опущены.

Дополняя (12) физическими соотношениями (7), условиями сопряжения оболочек и однородными граничными условиями, получим линейную однородную краевую задачу по определению величины критических осесимметричных нагрузок, действующих на 
составную оболочечную конструкцию. Докритическое изменение геометрии оболочек учитывается в результате записи уравнений (11), (12) для геометрии докритического деформированного осесимметричного состояния оболочки.

Однородные линейные краевые задачи по определению критических нагрузок для составных оболочечных конструкций, полученные выше, решаются на основе алгоритма, изложенного в работе [10].

Признаком выпучивания оболочки по k-й гармонике является достижение такого уровня нагрузки, при котором решение однородной линейной краевой задачи отличается от тривиального (нулевого). За критическую нагрузку принимается минимальная из нагрузок, найденных для каждого номера гармоники.

Представим общее решение линейной однородной задачи (11) для любой k-й гармоники в форме

$$
\bar{N}(t)=\sum_{i=1}^{4} C_{i} \bar{N}_{i}(t),
$$

где $\bar{N}_{i}(t)$ - общие решения однородной линейной задачи.

Однородные граничные условия на правом крае имеют вид:

$$
B_{2} \bar{N}=0 \text { при } t=t_{N},
$$

где $B_{2}$ - матрица порядка $4 \mathrm{x} 8 ; \bar{N}$ - вектор-столбец 8-го порядка.

Подставляя общее решение (13) в граничные условия (14), получим однородную систему линейных уравнений для определения постоянных $C_{j}$.

$$
\sum_{j=1}^{4} a_{i j} C_{j}=0, \quad(i=1,2,3,4) .
$$

Существование нетривиального решения задачи связано с наличием ненулевого решения системы (15), которое возможно при равенстве нулю ее определителя.

Таким образом, уравнение устойчивости по определению критической нагрузки для k-й гармоники имеет вид:

$$
\left|a_{i j}^{(k)}\right|=0
$$

Записать уравнение (16) в явном виде не представляется возможным.

Введем безразмерный параметр $\eta$, характеризующий уровень обобщенной нагрузки $P$ :

$$
\eta=\frac{P}{P_{0}}
$$

где $P_{0}-$ некоторое постоянное значение нагрузки.

Вычисляя значение определителя (16) с некоторого начального значения параметра $\eta_{0}$ с шагом $\Delta \eta$, находим для каждого номера гармоники критическое значение параметра $\eta_{\kappa p}$, при котором определитель обращается в ноль. По наименьшему из всех найденных критических значений - критическую нагрузку. Для установленной критической нагрузки и номера гармоники можно определить форму потери устойчивости [13], которая может быть использована при проектировании оболочечной конструкции.

По изложенному выше алгоритму была составлена программа по отысканию величин критических осесимметричных нагрузок и форм выпучивания для составных оболочечных конструкций при сильном изгибе. 
open-aceess journal

\section{3. Исследование устойчивости гибких сферических панелей}

Рассмотрим устойчивость сферической панели, нагруженной внешним давлением q (рис. 1). Панель имеет радиус кривизны $\mathrm{R}=516,5 \mathrm{Mм}$, радиус контура $\mathrm{r}=100 \mathrm{мм}$, постоянную толщину в полюсе $\mathrm{h}_{0}=1 \mathrm{mм}$, модуль упругости материала $\mathrm{E}=200$ ГПа, коэффициент Пуассона $v=0,3$. Толщина оболочки вдоль образующей изменяется по линейному закону, и на внешнем крае $\mathrm{h}_{1}$ изменяется в пределах: от 0,7 мм до 1,3 мм. Точность решения задачи $\varepsilon=0,0001$.

На рис. 2 приведены величины критической нагрузки в зависимости от толщины оболочки на внешнем крае $\mathrm{h}_{1}$ и условий закрепления: 1 -защемление; 2 -шарнирнонеподвижное закрепление; 3 - шарнирно-подвижное закрепление; 4 - шарнирно-подвижное закрепление с опорным кольцом (размеры поперечного сечения $4 \times 4$ мм). В таблице представлены численные значения критических нагрузок $\mathrm{q}_{\text {кр }}$ и количество волн по окружности при потере устойчивости $n$.

Из зависимостей следует, что при увеличении толщины контура оболочки величина критической нагрузки увеличивается, а при увеличении податливости опор снижается.

Формы потери устойчивости оболочки постоянной толщины ( $\mathrm{h}=1 \mathrm{Mм})$ показаны на рис. 3 и представляют собой изменение относительного прогиба $\bar{w}=w / w_{\max }$ вдоль образующей оболочки.

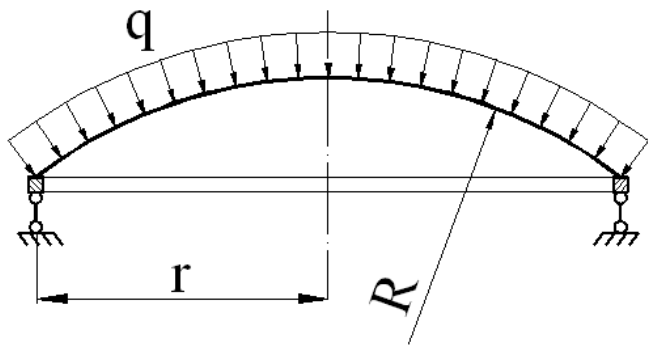

Рис. 1. Схема оболочки

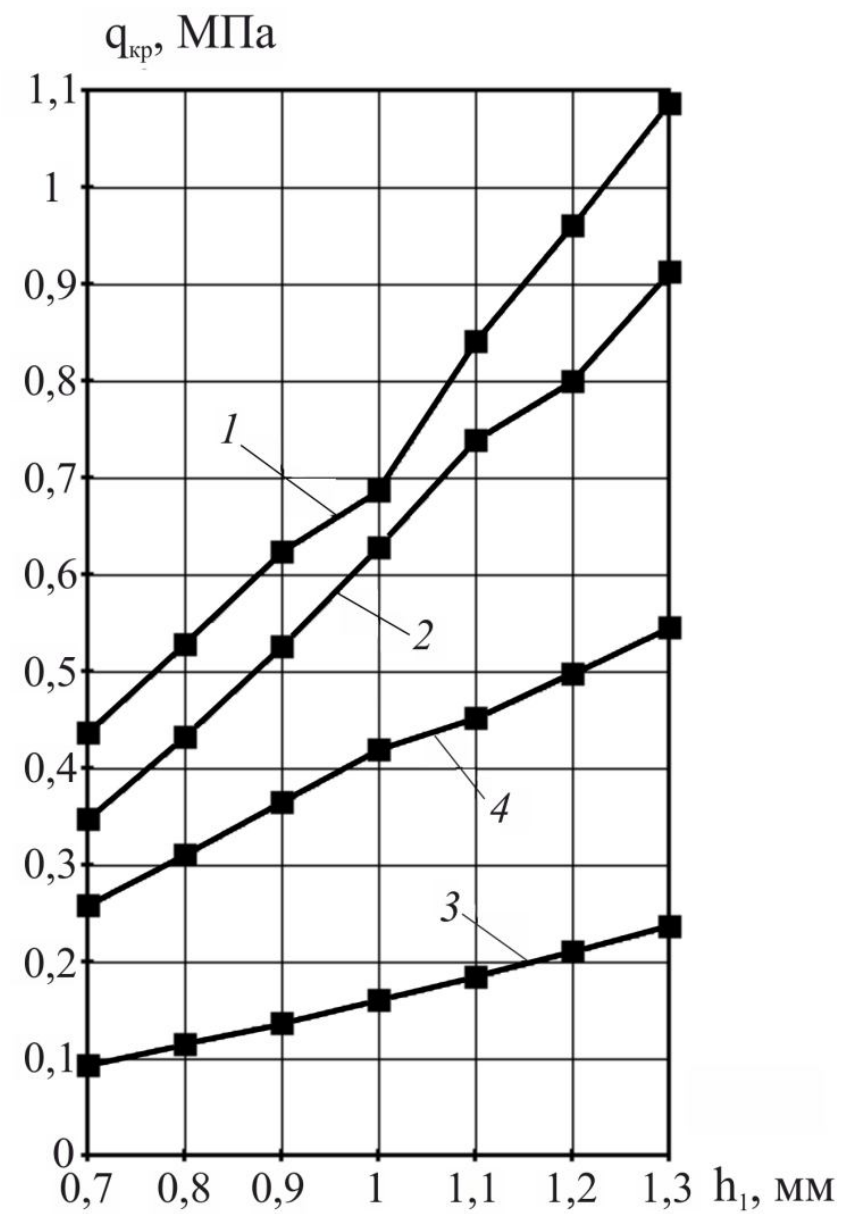

Рис. 2. Зависимость критической нагрузки от толщины на внешнем крае 
Численные значения критических нагрузок и количество волн по окружности при потере устойчивости

\begin{tabular}{|c|c|c|c|c|c|c|c|}
\hline $\mathrm{h}_{1}, \mathrm{MM}$ & 0,7 & 0,8 & 0,9 & 1,0 & 1,1 & 1,2 & 1,3 \\
\hline \multicolumn{8}{|c|}{ Оболочка с защемленным краем } \\
\hline $\mathrm{n}$ & 5 & 5 & 5 & 4 & 5 & 5 & 5 \\
\hline $\mathrm{q}_{\text {кр}}, \mathrm{MПа}$ & 0,438 & 0,528 & 0,625 & 0,688 & 0,841 & 0,960 & 1,087 \\
\hline \multicolumn{8}{|c|}{ Шарнирно-неподвижное закрепление } \\
\hline $\mathrm{n}$ & 6 & 6 & 6 & 6 & 6 & 5 & 5 \\
\hline $\mathrm{q}_{\mathrm{кр}}, \mathrm{MПа}$ & 0,348 & 0,432 & 0,525 & 0,627 & 0,738 & 0,801 & 0,913 \\
\hline \multicolumn{8}{|c|}{ Шарнирно-подвижное закрепление } \\
\hline $\mathrm{n}$ & 3 & 3 & 3 & 3 & 3 & 3 & 3 \\
\hline $\mathrm{q}_{\mathrm{kp}}, \mathrm{M \Pi а}$ & 0,0933 & 0,114 & 0,137 & 0,160 & 0,185 & 0,210 & 0,236 \\
\hline \multicolumn{8}{|c|}{ Шарнирно-подвижное закрепление с опорным кольцом 4×4 мм } \\
\hline $\mathrm{n}$ & 4 & 4 & 4 & 4 & 3 & 3 & 3 \\
\hline $\mathrm{q}_{\mathrm{кр}}, \mathrm{M \Pi а}$ & 0,259 & 0,311 & 0,364 & 0,419 & 0,451 & 0,498 & 0,545 \\
\hline
\end{tabular}

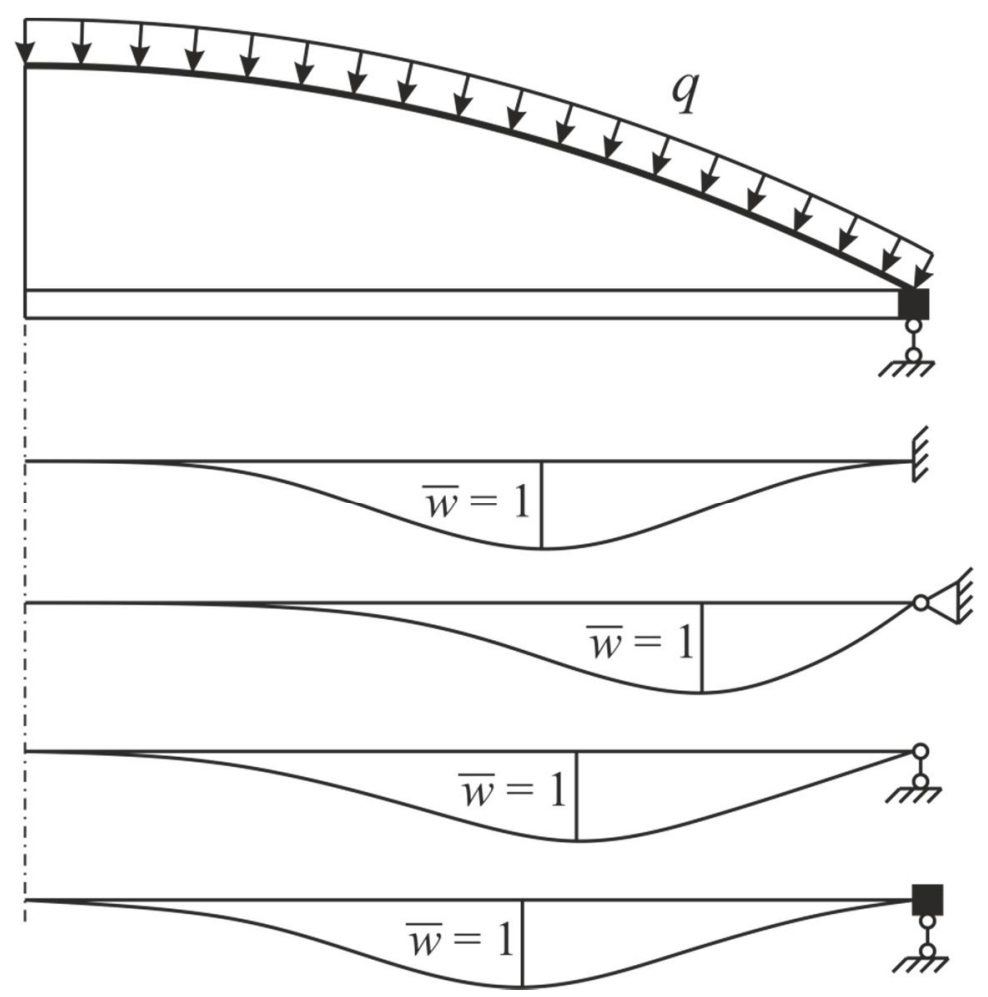

Рис. 3. Формы потери устойчивости

На рис. 4 представлены кривые деформирования защемленной по контуру оболочки в координатах давление $\mathrm{q}-$ прогиб полюса $\mathrm{w}_{0}: 1$ - постоянной толщины $\mathrm{h}_{0}=1 \mathrm{мм}[16] ; 2-$ переменной толщины, изменяющейся по линейному закону вдоль образующей; 3 переменной толщины, изменяющейся вдоль образующей по закону квадратной параболы $h(s)=h_{0}+\left(h_{1}-h_{0}\right) \cdot\left(\frac{s-s_{0}}{s_{1}-s_{0}}\right)^{2} ; 4-$ переменной толщины, изменяющейся вдоль образующей по экспоненциальному закону $h(s)=h_{0}\left(\frac{h_{1}}{h_{0}}\right)^{\left(\frac{s-s_{0}}{s_{1}-s_{0}}\right)^{2}}$. Толщина оболочки на контуре $\mathrm{h}_{1}=3$ мм.

Оболочка постоянной толщины (кривая 1) теряет устойчивость до первой предельной 
точки с образованием четырех волн по окружности при давлении $\mathrm{q}^{4}$ кр $=0,688$ МПа. Оболочки переменной толщины выворачиваются осесимметрично без перехода на неосесимметричные формы равновесия (кривые 2, 3 и 4).

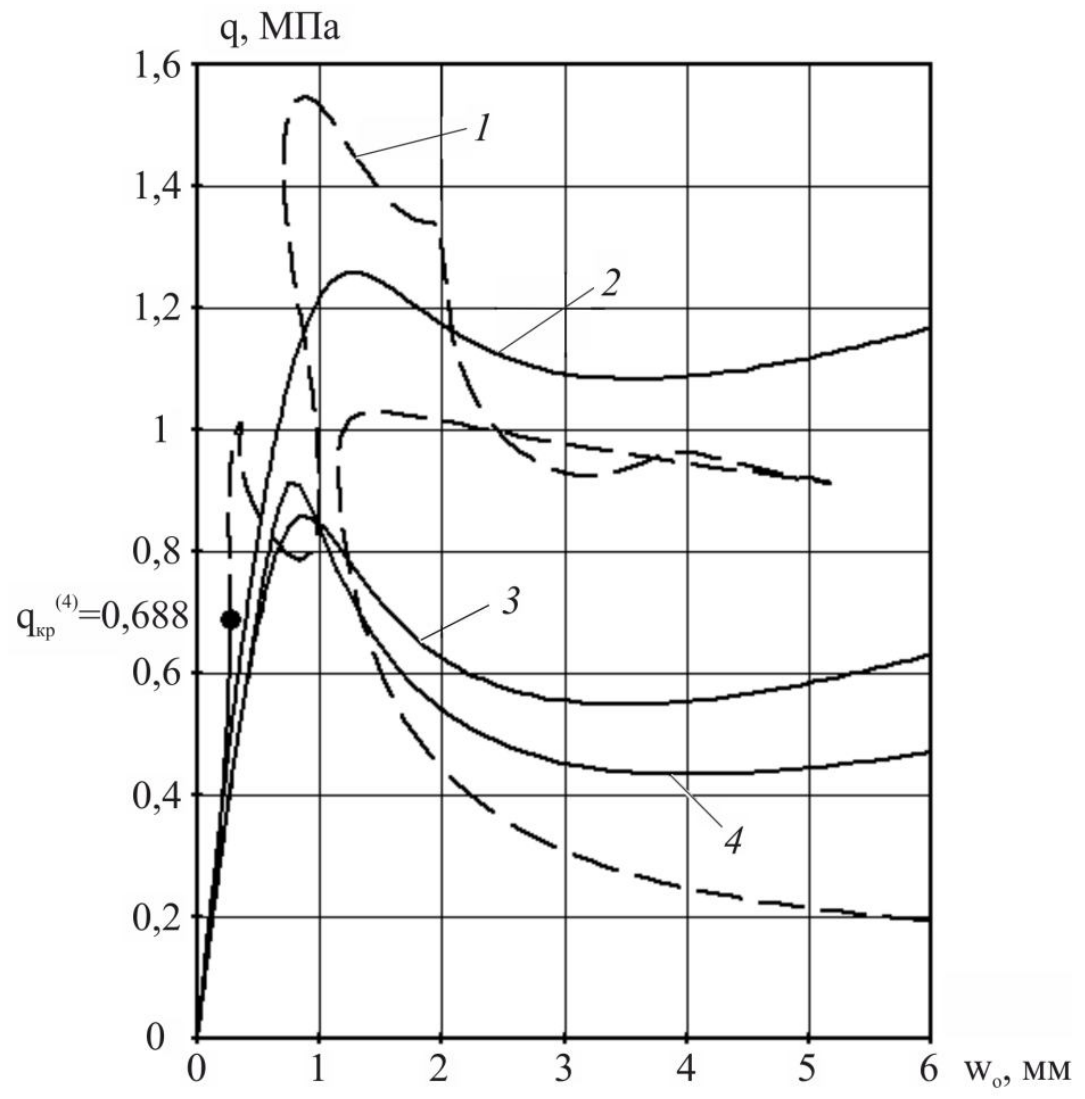

Рис. 4. Кривые деформирования оболочки

Таким образом, использованный подход позволяет исследовать устойчивость составных оболочечных систем с хорошей степенью точности.

\section{Литература}

1. Валишвили Н. В. Методы расчета оболочек вращения на ЭЦВМ. - М. : Машиностроение, 1976. - 278 с.

2. Вольмир А. С. Устойчивость деформируемых систем. - М. : Наука, 1976. - 984 с.

3. Годунов С. К. О численном решении краевых задач для систем обыкновенных линейных дифференциальных уравнений // Успехи математических наук. - 1961. - Т. 16, вып. 3. - С. 171-174.

4. Григолюк Э. И., Шалашилин В. И. Проблемы нелинейного деформирования. - М. : Наука, 1988. - 231 с.

5. Григоренко Я. М. Изотропные и анизотропные слоистые оболочки вращения переменной жесткости. - Киев : Наукова думка, 1973. - 228 с.

6. Grigorenko Ya. M., Kryukov N. N. Solving nonlinear boundary-value problems of the statics of flexible laminated shells in the supercritical region // Soviet Applied Mechanics. - 1983. Vol. 19, iss. 3. - P. 217-221.

7. Емельянов И. Г. Контактные задачи теории оболочек. - Екатеринбург : УрО РАН, 2009. - 184 c.

8. Канторович Л. В., Акилов Г. Р. Функциональный анализ в нормированных пространствах. М. : Физматгиз, 1959. - 684 с. 
9. Статика и динамика тонкостенных оболочечных конструкций / А. В. Кармишин, В. А. Лясковец, В. И. Мяченков, А. Н. Фролов. - М. : Машиностроение, 1975. - 376 с.

10. Климанов В. И., Чупин В. В. Статика и устойчивость гибких неоднородных оболочечных систем. - Красноярск : КрасГУ, 1986. - 182 с.

11. Коровайцев А. В. Об одном алгоритме исследования состояния непологих оболочек вращения при больших осесимметричных перемещениях // Изв. вузов. Машиностроение. 1981. - № 10. - C.12-15.

12. Муштари Х. М., Галимов К. З. Нелинейная теория упругих оболочек. - Казань : Таткнигоиздат, 1957. - $431 \mathrm{c.}$

13. Мяченков В. И., Григорьев И. В. Расчет оболочечных конструкций на ЭВМ. Справочник. - М. : Машиностроение, 1981. - 216 с.

14. Новожилов В. В. Основы нелинейной теории упругости. - М. : Гостехиздат, 1948. $211 \mathrm{c}$.

15. Рейсснер Е. Линейная и нелинейная теория оболочек // Тонкостенные оболочечные конструкции. - М. : Машиностроение, 1980. - С. 55-69.

16. Чупин В. В., Черногубов Д. Е. Устойчивость упругих составных оболочечных конструкций при осесимметричном нагружении // Строительство и образование: сборник научных трудов. - Екатеринбург : УрФУ, 2011. - Вып 14. - С. 29-32.

17. Shapovalov L. A. Equations of thin elastic shells for non-symmetric deformations // Mechanics of Solids. - 1976. - Iss. 3 - P. 62-72. 